\title{
Analysis and Research on the Timeliness of Virtual Reality Sports Actions in Football Scenes
}

\author{
Qingwen Tan (D) and Seung-Soo Baek (iD) \\ Department of Sport \& Health Science, College of Natural Science, Sangmyung University, Seoul 03016, Republic of Korea \\ Correspondence should be addressed to Seung-Soo Baek; 2009030226@st.btbu.edu.cn
}

Received 28 September 2021; Revised 26 October 2021; Accepted 27 October 2021; Published 19 November 2021

Academic Editor: Xin Ning

Copyright (C) 2021 Qingwen Tan and Seung-Soo Baek. This is an open access article distributed under the Creative Commons Attribution License, which permits unrestricted use, distribution, and reproduction in any medium, provided the original work is properly cited.

In the complex and changing situation on the soccer field, players must always be aware of their teammates, opponents, and the position of the ball during the game, constantly updating and analyzing the strategic information of the opponent in order to make appropriate tactical decisions. This ability to track multiple objects at the same time is also a prerequisite for high-level soccer players to be able to react quickly and appropriately during the game. Therefore, it is essential to examine the attentional ability of soccer players in dynamic scenarios. This study compares soccer players' performance in 2D planar and 3D virtual reality dynamic tracking tasks in two dimensions. They are correct tracking rate and tracking speed. This paper examines the tracking performance and spatial attention allocation characteristics of soccer players in different dynamic tracking tasks and the differences with the average college students by manipulating different types of $2 \mathrm{D}$ dynamic tracking tasks and incorporating a point detection paradigm. It was found that there were no differences in correct tracking and detection stimulus awareness between soccer players and college students in different $2 \mathrm{D}$ dynamic tracking tasks, showing consistency across populations. In terms of correct tracking rates, both soccer players and university students showed the highest correct tracking rates in the location MIT task, followed by the MOT task, and the worst in the identity MIT task. This indicates that the good dynamic attention ability of soccer players was not reflected in the above 2D dynamic tracking process. However, soccer players and college students showed consistent characteristics across populations in different dynamic tracking tasks. The results of detection stimulus awareness showed that soccer players and college students had the same trend of attention allocation between dynamic tracking tasks, i.e., more attention to the blank area of the screen and the target object and less attention to the distractor. This suggests that there was a distractor suppression effect between different dynamic tracking tasks.

\section{Introduction}

The ever-changing living environment requires people to pay constant attention to the changing information in the visual field. For example, drivers need to keep an eye on traffic lights, vehicles, and pedestrians coming and going in front and behind them to ensure driving safety [1]. Therefore, this ability to track multiple objects of motion simultaneously is crucial to our daily life. In addition, as a high-speed, strongconfrontation, and multivariation sport, soccer players must always pay attention to the direction of the ball and the position of teammates and opponents during the game in order to quickly obtain the time and space information on the field and make appropriate action responses [2]. Therefore, it is essential to understand and examine the visual attention ability of soccer players in dynamic scenarios. In laboratory studies, dynamic visual tracking ability has been examined mainly through the multitarget tracking paradigm, in which the position (and identity) of multiple motor objects is continuously attended to and memorized over a period of time in order to maintain coherent perception of dynamic situations [3]. The classical MOT paradigm consists of three main phases: first, in the target-tagging phase, a number of twodimensional planar objects (about 6-10) with identical surface features are presented on the computer screen, and then some of them (about 3-5) are tagged as targets by means of 
flickering, and the remaining untagged objects are called distractors [4]. During the tracking phase, the flashing disappeared and all the objects moved on the screen, at which point the participants were asked to track the objects that had flashed during the target marking phase in real time. In addition, the speed of movement of the objects (viewing angle $1-15^{\circ}$ ) and the mode of movement (random or linear movement) can be adjusted according to the different research questions [5]. During the response phase, all objects stopped moving and participants were asked either to select all targets tracked (holistic reporting method) or to determine whether a probe object was a target. Numerous studies have shown that participants are able to track 4-5 targets simultaneously with a correct rate of $85 \%-95 \%$.

Virtual reality (VR) technology is mainly through computer technology to simulate the real environment and the use of computer three-dimensional image technology, simulation technology, sensing technology, display technology, and other external equipment, so that individuals perceive or experience the realistic three-dimensional visual, auditory, tactile, taste, and smell, and other senses [6]. Through the virtual reality system, individuals can interact with the virtual environment in real time and efficiently and create a sense of immersion. This reflects the three main characteristics of virtual reality technology, namely, multisensory, immersion, and interactivity. Immersion is an important indicator of the degree of experience and realism of a virtual environment. It refers to the degree to which participants feel that they are in a computer-simulated environment and have the same perceptual experience as if they were in a real environment, i.e., the degree to which individuals react to the virtual environment or the subjective feeling of being in the environment. There are two concepts related to immersion: immersion and immersion. Among them, immersion refers to the extent to which virtual reality technology is able to present participants with a broad, vivid visual scene [7]. It can be influenced by the size of the field of view, display size, stereo vision, the number of sensory systems simulated by the virtual system, the realism of the displayed stimuli, and other factors. And immersion is a psychological state or subjective feeling, i.e., the psychological feeling of the participant being in the virtual environment. It can be used as an indicator of the consistency of participants' reactions and emotions in real and virtual situations and can be measured in different ways (e.g., physiological responses, behavioral measures, and self-assessments). In short, immersion is an objective property of VR systems and an objective assessment of the level of technology they have achieved, an evaluation of the detail and realism of the virtual environment. Immersion, on the other hand, describes the psychological response of the participant to the virtual environment. With the development of technology, virtual reality systems have been divided into desktop, immersive, distributed, and other types. Among them, immersive virtual reality systems have become one of the most researched and applied in recent years due to their higher immersion [8]. At present, the immersive virtual environment technology mainly includes two types: one is a cave-like virtual reality system. This system mainly projects visual images generated by computer technology onto the translucent walls, floor, and ceiling of a three-dimensional cube room in which individuals can move freely and multiple stereoscopic projection screens and speakers are placed around the individuals. With the help of stereoscopic glasses and other sensing devices, the individual can not only generate stereoscopic vision but also touch and change the objects in the environment. The second and more common is the use of head-mounted display (HMD) systems to present virtual environments. This system connects a computer to a head motion tracking device, which is used to measure the position and orientation of an individual's head in the physical environment and transmits this information to the computer in real time to build a three-dimensional representation of the virtual environment [9]. At any given moment, the computer system generates visual and auditory images based on the position and orientation of the individual's head and outputs them to the head-mounted display [10]. The virtual scene presented by the head-mounted display provides cues such as binocular parallax, convergence, and retinal parallax, thus enabling the individual to develop a self-centered depth perception that brings a high level of immersion [11].

The current study focuses on deepening the understanding of tracking performance and attention allocation characteristics among different $2 \mathrm{D}$ dynamic tracking tasks, comparing the differences in performance between $2 \mathrm{D}$ and $3 \mathrm{D}$ dynamic tracking tasks, and analyzing the sensitivity and validity of the dependent variable metrics of tracking correctness and tracking speed. Specifically, the following points are covered: (1) further examination of tracking performance and influencing factors in different dynamic tracking tasks. The differences in tracking performance between different dynamic tracking tasks were compared on the one hand, and the differences in attention allocation characteristics between different dynamic tracking tasks were compared on the other hand, in an attempt to explain the characteristics of attention allocation in the dynamic tracking process. (2) Extending the dynamic tracking task from a $2 \mathrm{D}$ plane to a $3 \mathrm{D}$ virtual reality scene to improve the ecological validity of the study and examining the relationship between 2D and 3D dynamic tracking tasks to explore the advantages of the 3D dynamic tracking task in dynamic attentional ability measurement. (3) To examine whether tracking speed is a more sensitive measure of individual differences compared to tracking correctness. This study mainly used the dynamic tracking paradigm and combined it with virtual reality technology to place the dynamic tracking task in a 3D space to make the task more consistent with the motion scenario, in order to provide experimental evidence and reference for the dynamic attentional processing characteristics of soccer players. In addition, the tracking performance of soccer players was further considered an indicator of athletes' dynamic visual attention ability, with a view to supporting test selection and training of athletes.

\section{Related Work}

At present, studies that have explored the MOT paradigm on dynamic visual attention have mainly focused on the influencing factors of tracking performance. A large number 
of studies have found that the number of target and distractor objects, surface features of objects, variation in object motion space, size of object spacing, speed of object motion, motion mode, and motion trajectory all affect multitarget tracking performance. In addition, some researchers have also explored the relationship between multitarget tracking and working memory and the role of limbic vision on multitarget tracking tasks [12]. Although a large number of studies have shown that tracking performance in MOT tasks can be affected by many of these factors, the role of attention in the tracking process is still very vague. In recent years, numerous studies have shown through behavioral, electrophysiological, and neuroimaging evidence that the multiobjective tracking task is an attentional resource-draining task. It was found that tracking performance on the MOT task suffered from broken loops when participants were asked to complete both the MOT task and a number of discrimination tasks (e.g., comparing the size of a number on a screen to a 5 and reporting it) [13]. Researchers also used a dual-task paradigm combining the MOT task with either a visual search task or an auditory discrimination task and found that the MOT task did not share attentional resources with the visual search task but occupied attentional resources with each other with the auditory discrimination task.

It has also been suggested that two tasks that both rely on visual input may cause confounding of visual attentional resources due to visual processing interactions, and to avoid interference between the two visual tasks, this study combined the MOT task and the pitch discrimination task [14]. It was found that the MOT task and the pitch discrimination task performed worse when completed simultaneously than when completed separately, suggesting that multitarget tracking requires attentional engagement. In addition, another study also found that listening to voice narration had no effect on tracking performance compared to tracking performance on a single MOT task, but telephone conversations disrupted tracking performance on the MOT task. This suggests that telephone conversations interfere with the central stage of information processing during tracking. Later, the researchers used a dual-task paradigm to examine the role of the MOT task in visual working memory [15]. The results revealed that the MOT task disrupted the performance of visual working memory, especially feature binding in working memory [16]. This suggests that the MOT task shares attentional resources with visual working memory. In summary, most explorations of the role of attention in the MOT task have used a dual-task paradigm in which the MOT task is combined with another subtask to examine the effect of the subtask on the performance of the multiobjective tracking task and then analyze the extent to which the two tasks occupy and deplete attentional resources. The results all found that the dual-task paradigm leads to a decrease in multiobjective tracking performance [17].

Thus, the multiobjective tracking task is one that requires depletion of attentional resources. During tracking, only the involvement of attention can maintain the continuity of the spatio-temporal characteristics of the object. Although the above studies consistently show that attention plays an important role in multitarget tracking tasks, there is controversy regarding the allocation of attention during tracking. So far, a dual-task paradigm has also been used for the allocation of attention during multitarget tracking, i.e., a combination of a multitarget tracking task and a dot detection task, which requires individuals to simultaneously perceive detection stimuli (e.g., small red dots and small squares) that appear at different locations on the screen (target, distractor, and blank area of the screen) during multitarget tracking [18]. The dot detection task hypothesizes that detection stimuli are more likely to be perceived when they appear in the area that the individual is currently attending to, even if the detection stimulus is not relevant to the task requirements. Some studies have found that in the MOT task, individuals allocated more attention to the tracked target; i.e., there was a target attention enhancement effect [19]. However, it has also been found that participants predominantly suppressed distractors during tracking; i.e., there was distractor suppression. In contrast, it has been shown that the purpose of individual inhibition of distractors is mainly to distinguish targets from distractors, and when targets and distractors cannot be distinguished in the preattentive stage, participants will inhibit distractor objects that are easily confused with targets during tracking to ensure accurate tracking of targets.

In addition, it has also been suggested that target activation and distractor inhibition may occur in parallel during tracking and that this attentional allocation feature is primarily related to task difficulty and varies flexibly with task demands (tracking load) [20]. When task difficulty is low, visual attention is not necessary for tracking task completion, but increasing task difficulty leads to competition for attention in both tracking and detection tasks. Recent studies have also shown that the allocation of attention during multitarget tracking is not only an automated process of the vision system but also a top-down, goal-driven process [21]. Overall, since MOT tasks usually use targets with identical surface features and distractors and targets and distractors can only be distinguished at the target marking stage, this makes it impossible for the observer to use the identity or surface features of the object during tracking and can only rely on the object's motion information or spatiotemporal characteristics (e.g., position, trajectory, velocity, and direction) to establish object continuity in order to maintain multiple motion object tracking. Therefore, the multitarget tracking process not only requires the involvement of attention but also consumes more attentional resources when the task difficulty increases. In addition, during multitarget tracking, visual attention is flexibly allocated according to the task requirements, and there may be both attentional activation for the target object and attentional suppression for the distractor, depending on the similarity between the target and the distractor (e.g., motion pattern, shape, or depth perception). In short, attention is not only a bottom-up, stimulus-driven process during tracking but also a top-down, target-driven process.

\section{Soccer Player Dynamic Tracking in 3D Virtual Reality}

3.1. Motion Simulation and Dynamic Analysis. The dynamic visual tracking tasks in this study were all tested using a Lenovo Legion R720-15IKBM computer. The experimental 
materials are shown in Figure 1. Depending on the dynamic tracking task, the stimulus materials were divided into $2 \mathrm{D}$ planar yellow solid circles (as shown in Figure 2) and 3D stereoscopic yellow spheres. Thus, in the 2D planar MOT task (hereafter referred to as PC2D), the stimulus material was a $2 \mathrm{D}$ yellow solid circle with a diameter of $1.7 \mathrm{~cm}$, the stimulus presentation area was a rectangular box of $30.6 \times 15.3 \mathrm{~cm}$ (43.6 for a horizontal viewing angle and $22.6^{\circ}$ for a vertical viewing angle), and the computer display was about $42 \mathrm{~cm}$ from the subject's eyes with a blue screen background. In the $3 \mathrm{D}$ virtual reality MOT task (hereafter referred to as VR3D), the motion object was a 3D yellow stereoscopic sphere, and the stimulus presentation area was a cubic room with white walls and blue floor. The distance from the headmounted display to the wall of the cube room is $4 \mathrm{~m}$. The cross section is made at the midpoint of the width of the cube room, and the size of the cross section is a $5 \times 2.5 \mathrm{~m}$ rectangle, and the view size of the rectangle is the same as the view size of the stimulus presentation area in the PC2D task. In addition, the initial velocity of the object in the PC2D task was $4.161 \mathrm{~cm} / \mathrm{s}\left(5.72^{\circ} / \mathrm{s}\right)$, while the initial velocity of the object in the VR3D task at the cross section of the cube space was the same as in the PC2D task, to ensure that the parameters of the PC2D task and the VR3D task were consistent.

A mixed design of 2 (task type : VR3D, PC2D) $\times 2$ (group: soccer players, general college students) was used in this study, where group was the between-group variable and task type was the within-group variable. The dependent variable was the tracking speed threshold, including three indicators: the threshold of the last four transitions (referred to as the last four thresholds), the average threshold, and the extreme value. Tracking speed is the value of object movement speed that participants can achieve when tracking the target object during the object movement phase. The threshold is the midpoint of the object motion velocity value corresponding to the correct and incorrect tracking trial transitions of the participant. In addition, the last four thresholds are the average of the participants' object motion velocities at the last four turning points (turns of correctly tracked and incorrectly tracked trials) during tracking. The mean threshold was the average of the subject's object motion velocity at all turn points (turns of correctly tracked trials and incorrectly tracked trials) throughout the tracking process. The extreme value is the maximum velocity of the object motion for all correct tracking trials of the participant.

3.2. Virtual Reality Model Detection. All participants completed the experiment independently in a quiet laboratory and were administered individually by the master test. The experimental procedure of the PC2D task is shown in Figure 3. First, 8 discs were presented on the computer screen as motion objects, 4 of which were marked as targets by flashing red and the remaining 4 discs were distractors, with a flashing time of $4 \mathrm{~s}$ (3 times); after the flashing stopped, all the discs started to move randomly and independently on the screen, with a motion duration of $8 \mathrm{~s}$ and an initial motion speed of $5.72 \%$ s. The subject's task was to track the target object; when the object motion stopped,

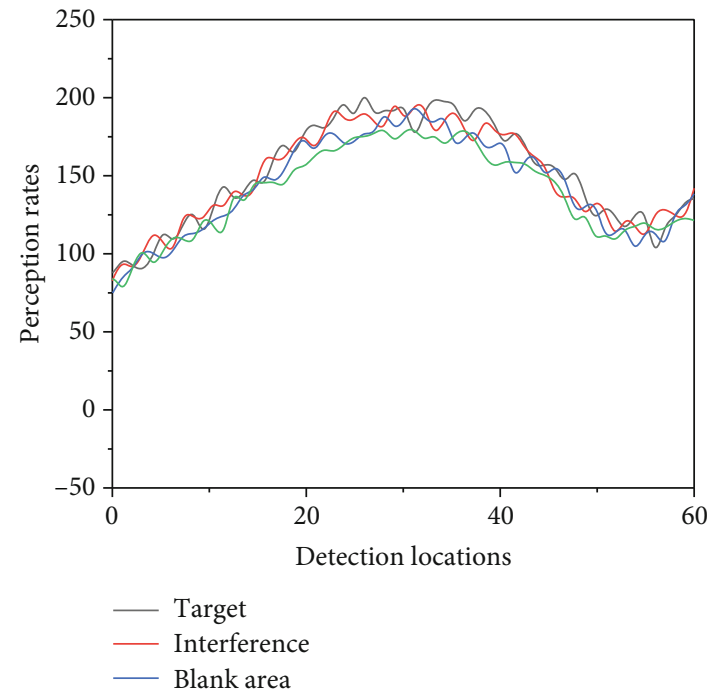

FIgURE 1: Detection stimulus perception rates for different tasks and detection locations.

the participants were asked to select the target object they were tracking with the mouse and give feedback, with the correct answer flashing purple and the wrong answer flashing green. In addition, the whole experimental process used an adaptive procedure to control the movement speed of the object; i.e., when the participant responded correctly in one trial, the movement speed of the object in the next trial would be adjusted upward by $30 \%$, while when the subject responded incorrectly, the movement speed of the object would be adjusted downward by $20 \%$. During movement, the objects randomly change their direction of motion when they collide with the edge of the screen or with each other. The process is the same as in the PC2D task except that the participant is required to use the VIVE joystick to select a target object to track. However, the objects in the VR3D task were $3 \mathrm{D}$ spheres that varied in size due to depth perception as they moved through the cubic space. After completing the VR3D task, all subjects were asked to fill out a survey about VR immersion and were asked to report the extent to which they immersed themselves in the virtual environment and ignored their surroundings based on the questions. Each experimental condition consisted of 30 trials, for a total of 60 trials. Participants were asked to take a 5-minute break for every 30 trials completed, and the experiment lasted approximately 30 minutes. Participants were required to complete 5 practice sessions before the formal experiment. To control for practice or fatigue effects, the order of all experimental conditions was balanced using the ABBA method. Participants' response data were automatically recorded by the experimental program, and the data were analyzed and processed by SPSS 22.0 after the experiment was completed.

To examine the degree of agreement between the PC2D and VR3D tasks for dynamic visual attention ability measures, this study performed a correlation analysis of the tracking speed thresholds for both of these tasks. The results are shown in Figure 4, where there was a significant positive correlation $(p<0.01)$ between the PC2D and VR3D tasks, in 


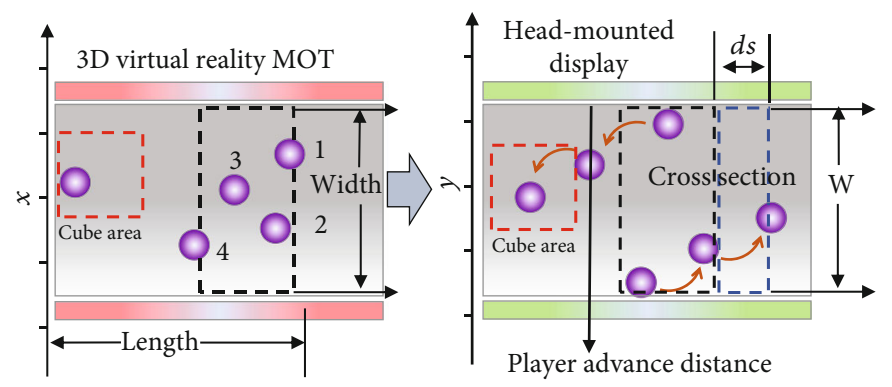

Figure 2: Dynamic visual tracking task.

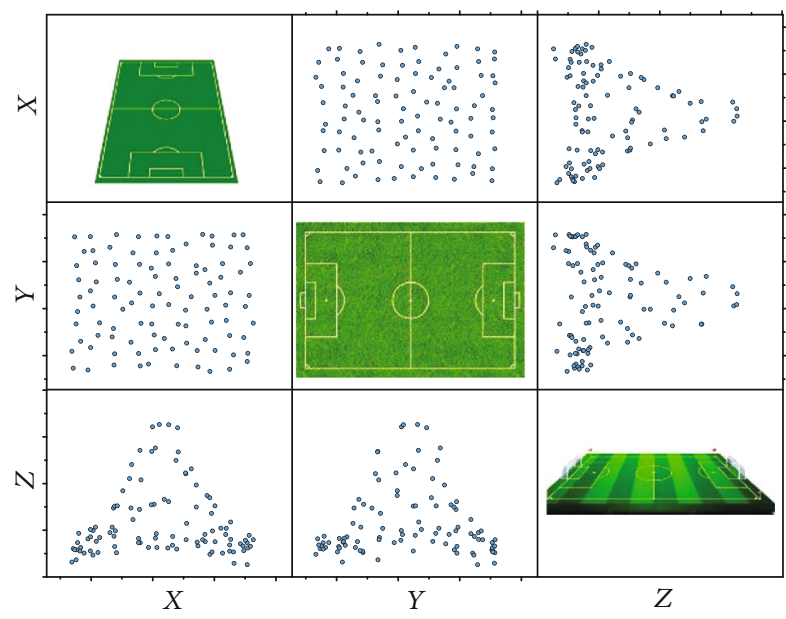

FIgURE 3: Virtual reality model detection.

terms of the last four thresholds, the mean threshold, and the extreme values. This indicates that the PC2D and VR3D tasks used in this study were in good agreement and both effectively measured the participants' dynamic visual attention abilities.

First, to exclude the effect of gender on group performance on tracking, a repeated measures ANOVA was conducted on gender and task type for general college students. The results showed that the main effect of gender was not significant at the last four thresholds $(F(1,46)=0.488, p=0.488)$. The interaction of gender with task type was not significant $(F(3,138)=1.186, p=0.317)$. At the mean threshold, the main effect of gender was not significant $(F(1,46)=0.423, p=0.519)$. The interaction of gender with task type was not significant $(F(3,138)=1.883)$. The interaction between gender and task type was not significant $(F(3,138)=1.883, p=0.153)$. At the extreme value, the main effect of gender was not significant $(F(3,138)=0.705$, $p=0.551)$. The interaction between gender and task type was not significant $(F(1,46)=0.204, p=0.656)$. In summary, it can be seen that there was no significant difference in tracking performance between male and female students, and the gender factor did not have an effect on tracking performance. Subsequently, a 2 (task type : VR3D, PC2D) $\times 2$ (group: soccer players, general college students) repeated measures ANOVA on the last four thresholds revealed a sig- nificant main effect of task type $(F(1,93)=21.543, p<0.001$, $\eta p 2=0.188$ ) (Greenhouse-Geisser correction). Further post hoc comparisons showed that the VR3D task tracked significantly faster than the PC2D task (1.60 vs. $1.37, p<0.001)$. The main effect of group was significant $(F(1,93)=18.702$, $p<0.001, \eta p 2=0.167)$. Football players tracked significantly faster than the average college student $(1.68>1.30, p<0.001)$. The interaction between task type and group was not significant $(F(1,93)=0.170, p=0.681)$.

3.3. Timeliness Analysis. A 2 (task type : VR3D, PC2D) $\times 2$ (group: soccer players, general college students) repeated measures ANOVA on the mean threshold found a significant main effect of task type $(F(1,93)=20.639, p<0.001$, $\eta p 2=0.182$ ) (Greenhouse-Geisser correction). The VR3D task had a tracking speed which was significantly higher than that of the PC2D task (1.45 vs. $1.30, p<0.001)$. The main effect of group was significant $(F(1,93)=17.446$, $p<0.001, \eta p 2=0.158)$. Football players tracked significantly faster than the average college student $(1.52>1.22$, $p<0.001)$. The interaction between task type and group was not significant $(F(1,93)=1.475, p=0.228)$. Specific ANOVA results are shown in Figure 5. Finally, a 2 (task type : VR3D, $\mathrm{PC} 2 \mathrm{D}) \times 2$ (group: soccer players, general college students) repeated measures ANOVA was performed on the extreme values. The results showed a significant main effect of task type $(F(1,93)=38.650, \quad p<0.001, \quad \eta p 2=0.294) \quad$ (GreenhouseGeisser correction). Further post hoc comparisons showed that the VR3D task tracked significantly faster than the PC2D task (2.00 vs. 1.72, $p<0.001)$. The main effect of group was significant $(F(1,93)=17.806, p<0.001, \eta p 2=0.161)$. Soccer players tracked significantly faster in the dynamic tracking task than the average college student $(2.06>1.66$, $p<0.001)$. This indicates significant differences in tracking performance between populations. The interaction between task type and group was significant $(F(1,93)=9.870, p=$ $0.002, \eta p 2=0.095)$. This suggests that tracking performance differs across populations in different task types.

A further simple effects test revealed a significant difference in tracking speed between soccer players and regular college students in the PC2D task $(F(1,93)=7.30, p=$ $0.008)$. Post hoc comparisons revealed that soccer players tracked faster than regular college students with a moderate effect size ( 1.84 vs. $1.59, p=0.008$, Cohen's $d=0.55)$. In addition to this, a significant difference in tracking speed between soccer players and average college students was also 


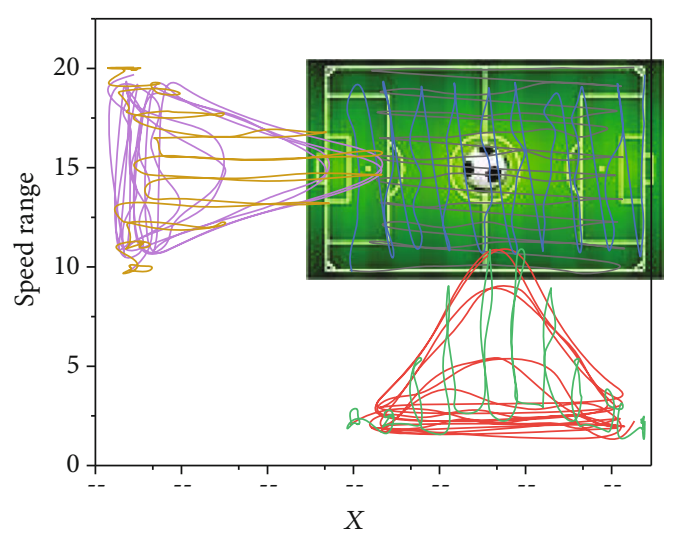

FIgURE 4: Tracking speed thresholds for VR3D tasks.

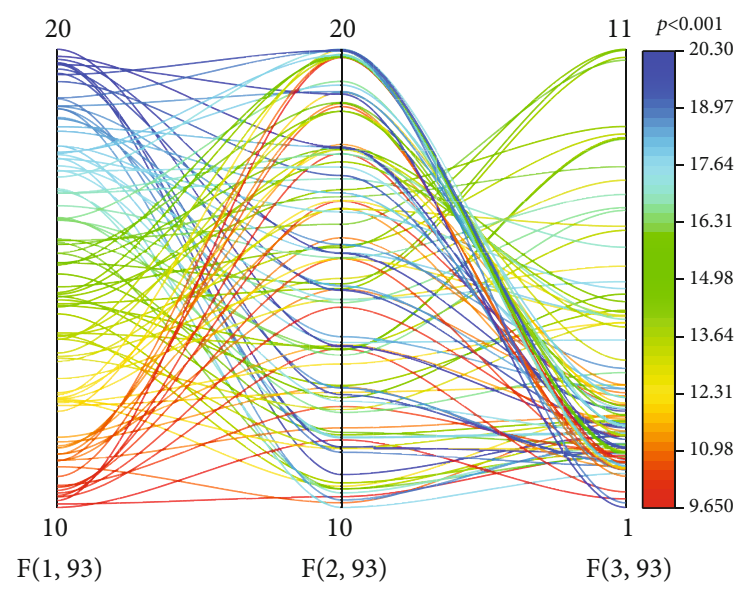

FIGURE 5: Variance of tracking speed thresholds for different task types.

found in the VR3D task $(F(1,93)=21.94, p<0.001)$, with soccer players tracking significantly faster than average college students with a large effect size (2.28 vs. $1.73, p<0.001$, Cohen's $d=0.96$ ). This suggests that soccer players tracked faster than average college students in both PC2D and VR3D tasks. To examine in depth whether the differences in tracking performance between soccer players and average college students in these two tasks were the same, the speed extremes of soccer players and average college students in the VR3D task were subtracted from their corresponding speed extremes in the PC2D task to obtain the corresponding VR3D-PC2D differences, and then, an independent samples $t$-test was conducted on the VR3D-PC2D differences of soccer players and average college students. It was found that the VR3D-PC2D difference was significantly higher for soccer players than for the average college student ( 0.44 vs. 0.15 , $t(93)=-3.127, p=0.002)$. This suggests that soccer players have a more significant tracking speed advantage in the VR3D task compared to the average college student.

\section{Functional Testing}

Overall, the tracking speed of the VR3D task was found to be significantly higher than that of the PC2D task, at the last four thresholds, the average threshold, and the extreme values. In addition, the tracking speed of soccer players was significantly higher than that of the average college student. In addition, the tracking speed extremes were found to be higher in both PC2D and VR3D tasks. The tracking speed of soccer players in the VR3D task was also faster than that of college students. A correlation test between soccer players' years in professional soccer clubs and the tracking speed thresholds in the PC2D and VR3D tasks revealed that soccer players' years in professional sports were only significantly and positively correlated with the mean threshold in the VR3D task $(r=0.320, p=0.014)$, and the correlation between each tracking speed threshold in the PC2D task was not significant ( $\mathrm{ps}>0.05$ ). This suggests that the longer the number of years a soccer player has been in a professional soccer club, the faster his or her tracking speed in the VR3D task (see Figure 6).

In addition, a further regression analysis of the correlation between the years of professional soccer players' entry into professional soccer clubs and the mean threshold of the VR3D task found that the athletes' years of professional sports explained $10.2 \%$ of the variance in the mean threshold of the VR3D task $(\beta=0.320, p=0.028)$. It can be seen that the years of professional sports experience of soccer players are more closely related to the VR3D task. The reason may lie in the fact that the longer the number of years a soccer player has been in a professional soccer club, the better his or her athletic experience or specialization, and this athletic expertise facilitates his or her tracking performance in the VR3D task. This study used virtual reality to present a dynamic tracking task in a $3 \mathrm{D}$ virtual space with tracking speed as the dependent variable indicator, as shown in Figure 7. The purpose was to examine whether soccer players had an advantage in the $3 \mathrm{D}$ virtual reality dynamic tracking task and to analyze the difference in their performance in the $2 \mathrm{D}$ and $3 \mathrm{D}$ dynamic tracking tasks. From the experimental results, it can be seen that the $3 \mathrm{D}$ virtual reality MOT task performs better than the 2D flat MOT task. In addition, compared with the results of experiment 1 , this study found that the tracking performance of soccer players was better than that of ordinary college students in both PC2D and VR3D tasks, and this advantage was even more obvious in the VR3D task. This also fully illustrates that (1) the observation of tracking speed is a more sensitive measure of soccer players' dynamic attention ability compared to the tracking correct rate used in experiment 1 , effectively differentiating individual differences. (2) Compared to the $2 \mathrm{D}$ dynamic tracking task, the $3 \mathrm{D}$ virtual reality tracking task effectively improved soccer players' tracking performance. This suggests that the $3 \mathrm{D}$ virtual reality task can better analyze the dynamic tracking ability of soccer players. Furthermore, the correlation between the professional sports years of soccer players and the mean threshold of the VR3D task also shows that the dominant effect of dynamic attentional tracking ability of soccer players is mainly manifested in the VR3D task.

To explore whether participants traded off responses to the tracking task versus the detection task, a correlation test 

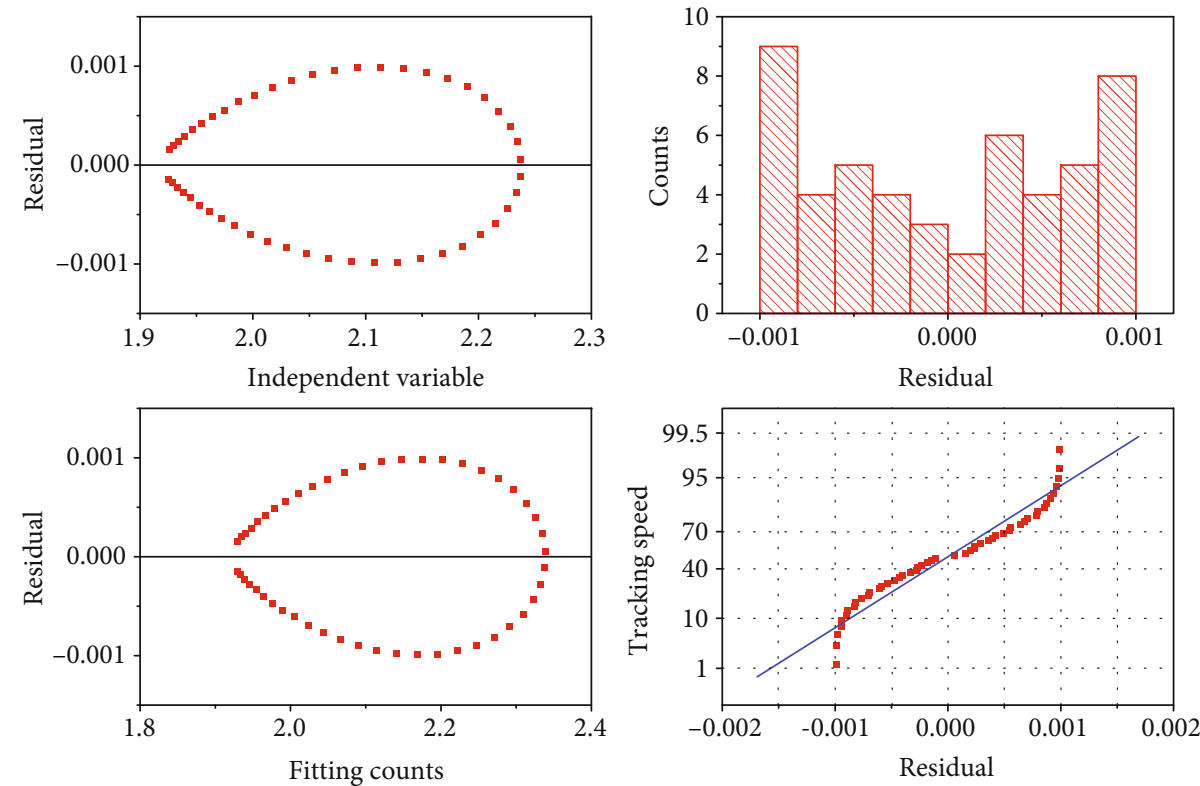

Figure 6: Correlation between years and tracking speed.

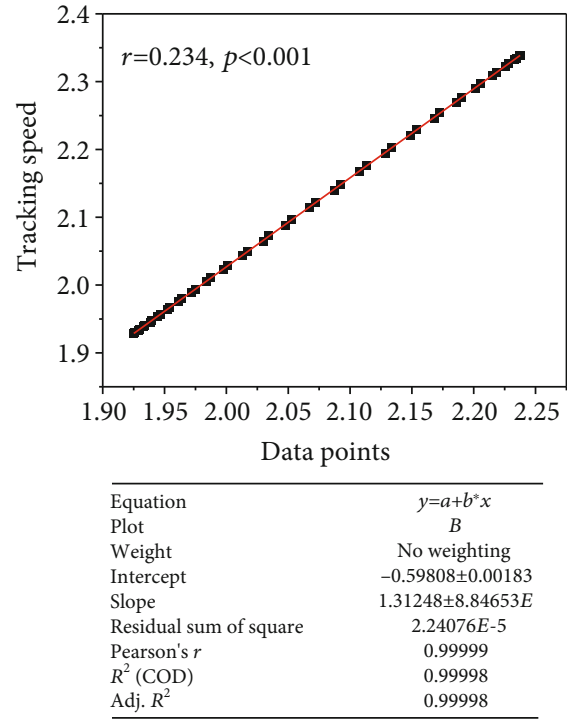

FIGURE 7: Tracking speed of different populations in different dynamic tracking tasks.

between the correct tracking rate and detection stimulus awareness rate revealed no correlation between the correct tracking rate $(M=79.22 \%, \mathrm{SD}=6.24 \%)$ and detection stimulus awareness rate $(M=64.30 \%, \mathrm{SD}=10.84 \%)(r=0.163$, $p=0.253)$. This suggests that the tracking task and the detection task are two separate tasks. In addition, due to the low detection stimulus perception rate in experiment 1 , the hit rate and false alarm rate of participants to the detection stimulus under different dynamic tracking tasks were analyzed in order to exclude the speculative nature of individuals to the detection stimulus. The results are shown in Figure 8 . This is mainly attributed to the improved ecological validity of the dynamic visual tracking task in $3 \mathrm{D}$ virtual

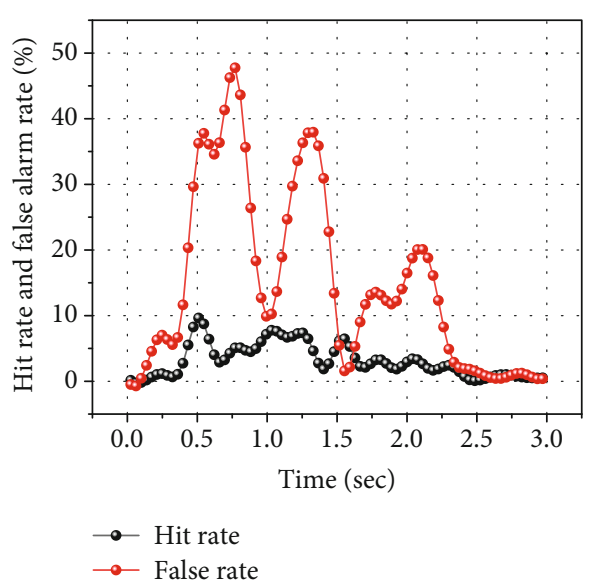

FIGURE 8: Hit rate and false alarm rate of detection stimuli.

reality scenes, which is more in line with the object tracking in realistic scenes, thus optimizing the efficiency of dynamic visual tracking. The hit rate of detection stimuli was higher than $50 \%$, and the false alarm rate was significantly lower than $50 \%$ in different dynamic tracking tasks, which indicates that the data of the detection perception rate in experiment 1 were valid and reliable.

In addition, to examine the effect of the false positive rate on the experimental results, a repeated measures ANOVA was conducted on the false positive rate for task type and group. It was found that the main effect of task type was not significant $(F(2,96)=0.735, p=0.482)$. The main effect of group was not significant $(F(1,48)=0.964, p=0.331)$. The interaction between task type and group was also not significant $(F(2,96)=0.529, p=0.591)$. In summary, the false alarm rates to the detection stimuli under different dynamic tracking tasks can be seen. There was no significant difference in the false alarm rate to the detection stimuli 
between soccer players and general college students. This indicates that the consistent false alarm rates under different tracking tasks and different populations did not have an effect on the results of detection perception rates. In summary, the detection stimulus awareness rate gradually decreased from MOT, location MIT, and then identity MIT tasks, and all of the above tasks showed a trend that the detection stimulus awareness rate was highest in the blank area of the screen, followed by the target, and lowest in the distractor (see Figure 1).

The results of tracking performance show that the tracking performance of the location MIT task is better than that of the MOT task, which in turn is better than that of the identity MIT task. This is consistent with the results of previous studies. In addition, the effect of detecting the location of stimulus appearance on the performance of different dynamic tracking tasks differed, but the effect was mainly found in the MOT and location MIT tasks and had no effect on the identity MIT task. In addition, in terms of the distribution of attention during tracking, the MOT task, the location MIT task, and the identity MIT task showed a trend of the highest detection stimulus awareness rate in the blank area of the screen, followed by the target and the lowest distractor. Notably, the present study subdivided the dynamic tracking task into the MOT task, location MIT task, and identity MIT task in an attempt to examine the tracking performance and attention allocation characteristics of soccer players in different tasks. This suggests that the soccer players did not show any difference in their dynamic visual attention ability and attention allocation characteristics in the $2 \mathrm{D}$ planar dynamic tracking task compared to the average college students. Therefore, given the dual-task paradigm used in experiment 1 , no differences in tracking performance and attention allocation characteristics were found between soccer players and college students. Moreover, most previous studies have used the MOT paradigm to examine athletes' dynamic attentional abilities; therefore, the follow-up experiment returned to a single task and examined soccer players' tracking performance using only the MOT paradigm.

\section{Conclusion}

This study will compare soccer players' performance in $2 \mathrm{D}$ planar and 3D virtual reality dynamic tracking tasks in terms of two dimensions: correct tracking rate and tracking speed. In particular, experiment 1 examined the tracking performance and attention allocation characteristics of soccer players in different dynamic tracking tasks by manipulating different types of traditional 2D dynamic tracking tasks in conjunction with a point detection paradigm and how they differed from the average college student. It was found that there was no significant difference between soccer players and university students in terms of both the correct tracking rate and detection stimulus perception rate in the dynamic visual tracking task. This indicates that the good dynamic attention ability of soccer players was not reflected in the above $2 \mathrm{D}$ dynamic tracking process. The results of the study found that there were no significant differences between soccer players and ordinary college students for either tracking performance between different dynamic tracking tasks or attention allocation characteristics during the tracking process. In addition, from the perspective of attention allocation, both soccer players and college students allocated more attention to the blank area of the screen and the target object and suppressed attention to distractors. And the 2D dynamic tracking task was used as a control condition to compare the differences between $2 \mathrm{D}$ and $3 \mathrm{D}$ tracking tasks. The results show that the VR3D task performs better than the PC2D task. In addition, the tracking performance of soccer players in both PC2D and VR3D tasks was better than that of college students, especially in the VR3D task. In addition, the longer the soccer player has been in the professional soccer club, the faster the tracking speed in the VR3D task.

\section{Data Availability}

The data used to support the findings of this study are included within the article.

\section{Conflicts of Interest}

All the authors do not have any possible conflicts of interest.

\section{References}

[1] M. Ashiri, B. Lithgow, A. Suleiman, B. Mansouri, and Z. Moussavi, "Quantitative measures of the visually evoked sensation of body movement in space (vection) using electrovestibulography (EVestG)," Virtual Reality, vol. 25, no. 3, pp. 731-744, 2021.

[2] R. Bahr, B. Clarsen, and J. Ekstrand, "Why we should focus on the burden of injuries and illnesses, not just their incidence," British Journal of Sports Medicine, vol. 52, no. 16, pp. 10181021, 2018.

[3] A. L. Borstad, R. Crawfis, K. Phillips et al., "In-home delivery of constraint-induced movement therapy via virtual reality gaming," Journal of Patient Centered Research and Reviews, vol. 5, no. 1, pp. 6-17, 2018.

[4] L. A. Cenydd and C. J. Headleand, "Movement modalities in virtual reality: a case study from ocean rift examining the best practices in accessibility, comfort, and immersion," IEEE Consumer Electronics Magazine, vol. 8, no. 1, pp. 30-35, 2019.

[5] A. Corsini, G. N. Bisciotti, C. Eirale, and P. Volpi, "Football cannot restart soon during the COVID-19 emergency! A critical perspective from the Italian experience and a call for action," British Journal of Sports Medicine, vol. 54, no. 20, pp. 1186-1187, 2020.

[6] C.-L. Deng, P. Geng, Y.-F. Hu, and S.-G. Kuai, "Beyond Fitts's law: a three-phase model predicts movement time to position an object in an immersive 3D virtual environment," Human Factors, vol. 61, no. 6, pp. 879-894, 2019.

[7] E. Depetris-Chauvin, R. Durante, and F. R. Campante, "Building nations through shared experiences: evidence from African football," The American Economic Review, vol. 110, no. 5, pp. 1572-1602, 2020.

[8] R. Grashow, M. G. Weisskopf, A. Baggish et al., "Premortem chronic traumatic encephalopathy diagnoses in professional football," Annals of Neurology, vol. 88, no. 1, pp. 106-112, 2020. 
[9] R. Grashow, M. G. Weisskopf, K. K. Miller et al., "Association of concussion symptoms with testosterone levels and erectile dysfunction in former professional US-style football players," Jama Neurology, vol. 76, no. 12, pp. 1428-1438, 2019.

[10] J. T. Johnston, B. R. Mandelbaum, D. Schub et al., "Video analysis of anterior cruciate ligament tears in professional American football athletes," American Journal of Sports Medicine, vol. 46, no. 4, pp. 862-868, 2018.

[11] A. Kittel, P. Larkin, N. Elsworthy, and M. Spittle, "Using $360^{\circ}$ virtual reality as a decision-making assessment tool in sport," Journal of Science and Medicine in Sport, vol. 22, no. 9, pp. 1049-1053, 2019.

[12] M. Marmot and J. Allen, "COVID-19: exposing and amplifying inequalities," Journal of Epidemiology and Community Health, vol. 74, no. 9, pp. 681-682, 2020.

[13] J. Mez, D. H. Daneshvar, B. Abdolmohammadi et al., "Duration of American football play and chronic traumatic encephalopathy," Annals of Neurology, vol. 87, no. 1, pp. 116-131, 2020.

[14] B. M. Mills, K. M. Conrick, S. Anderson et al., "Consensus recommendations on the prehospital care of the injured athlete with a suspected catastrophic cervical spine injury," Journal of Athletic Training, vol. 55, no. 6, pp. 563-572, 2020.

[15] F. Plessow, A. Pascual-Leone, C. M. McCracken et al., "Selfreported cognitive function and mental health diagnoses among former professional American-style football players," Journal of Neurotrauma, vol. 37, no. 8, pp. 1021-1028, 2020.

[16] A. L. Roberts, A. Pascual-Leone, F. E. Speizer et al., "Exposure to American football and neuropsychiatric health in former National Football League players: findings from the Football Players Health Study," American Journal of Sports Medicine, vol. 47, no. 12, pp. 2871-2880, 2019.

[17] H. Sarmento, F. M. Clemente, D. Araújo, K. Davids, A. McRobert, and A. Figueiredo, "What performance analysts need to know about research trends in association football (2012-2016): a systematic review," Sports Medicine, vol. 48, no. 4, pp. 799-836, 2018.

[18] K. Sarmiento, K. E. Thomas, J. Daugherty et al., "Emergency department visits for sports- and recreation-related traumatic brain injuries among children-United States, 2010-2016," Morbidity and Mortality Weekly Report, vol. 68, no. 10, pp. 237-242, 2019.

[19] R. A. Stern, C. H. Adler, K. Chen et al., "Tau positron-emission tomography in former National Football League players," The New England Journal of Medicine, vol. 380, no. 18, pp. 17161725, 2019.

[20] B. Thatcher, G. Ivanov, M. Szerovay, and G. Mills, "Virtual reality technology in football coaching: barriers and opportunities," International Sport Coaching Journal, vol. 8, no. 2, pp. 234-243, 2021.

[21] R. Yurko, S. Ventura, and M. Horowitz, "nflWAR: a reproducible method for offensive player evaluation in football," Journal of Quantitative Analysis in Sports, vol. 15, no. 3, pp. 163-183, 2019. 